\title{
EDIFICATE
}

I Congreso de Escuelas de Edificación y Arquitectura Técnica de España València, 4 y 5 de noviembre de 2021

Escuela Técnica Superior de Ingeniería de Edificación

Universitat Politècnica de València

Doi: https://doi.org/10.4995/EDIFICATE2021.2021.13511

\section{Convalidación de la formación en materia de Prevención de Riesgos Laborales del Sector de la Construcción establecida en el Convenio a los titulados de las Escuelas de Edificación y Arquitectura Técnica de España}

\section{Validation of the training in Occupational Risk Prevention in the Construction Sector established in the Agreement for graduates of the Spanish Schools of Building and Technical Architecture.}

\author{
María Segarra Cañamares ${ }^{a}$
}

aEscuela Politécnica de Cuenca. Universidad de Castilla-La Mancha. Maria.segarra@uclm.es

\begin{abstract}
Article 146 of the General Collective Bargaining Agreement for the construction sector, on training provided in university degrees, establishes that workers who hold university degrees which provide training in occupational risk prevention equivalent to that specified in this Agreement will have it recognized, as appropriate in each case, by agreement of the Board of Trustees of the Construction Labor Foundation.

Graduates of the Spanish Schools of Building and Technical Architecture receive training in occupational risk prevention that exceeds in content and competences that established in the agreement, which should lead to a direct and generally agreed validation, for all our Schools, of the Second cycle of training in Occupational Risk Prevention in the Construction Sector: Training by work post and the Basic Level of prevention in construction.
\end{abstract}

Keywords: Risk prevention training, construction, university, validation.

\section{Resumen}

El Convenio colectivo general del sector de la construcción establece en su artículo 146, sobre la formación impartida en títulos universitarios, que los trabajadores que estén en posesión de títulos universitarios en los que se imparta una formación en materia de prevención de riesgos laborales equivalente a la especificada en el presente Convenio, se procederá a su 
Convalidación de la formación en materia de Prevención de Riesgos Laborales del Sector de la Construcción establecida en el Convenio a los titulados de las Escuelas de Edificación y Arquitectura Técnica de España - Validation of the training in Occupational Risk Prevention in the Construction Sector established in the Agreement for graduates of the Spanish Schools of Building and Technical Architecture.

convalidación, según proceda en cada caso, mediante acuerdo del Patronato de la Fundación Laboral de la Construcción.

Los titulados de las Escuelas de Edificación y Arquitectura Técnica de España reciben una formación en prevención de riesgos laborales que excede en contenido y competencias la establecida en convenio, lo que debería suponer una convalidación directa y acordada, con carácter general, para todas nuestra Escuelas, del Segundo ciclo de formación en materia de Prevención de Riesgos Laborales del Sector de la Construcción: Formación por puesto de trabajo y del Nivel básico de prevención en construcción.

Palabras clave: Formación en prevención de riesgos, construcción, universidad, convalidación. 


\section{Introducción}

El Convenio colectivo general del sector de la construcción (CCGSC) (RDGE, de 21 de septiembre) establece en su artículo 146, sobre la formación impartida en títulos universitarios, que los trabajadores que estén en posesión de títulos universitarios en los que se imparta una formación en materia de prevención de riesgos laborales equivalente a la especificada en el presente Convenio, se procederá a su convalidación, según proceda en cada caso, mediante acuerdo del Patronato de la Fundación Laboral de la Construcción (FLC).

Actualmente algunas escuelas han optado por realizar convenios con la FLC, para que la formación impartida en la titulación le sea reconocida por la correspondiente al nivel básico de prevención en la construcción, a los efectos previstos en el CCGSC.

Dichos convenios establecen la necesidad de que los contenidos de las asignaturas se correspondan con los establecidos en el convenio, sin margen alguno a que dichos contenidos se impartan bajo otros epígrafes distintos a los indicados. Esto obligaría a las escuelas a tener que adaptar sus epígrafes a los marcados en el convenio, teniendo por tanto que modificar sus guías docentes, con lo que esto supone a efectos de la planificación y programación de unas asignaturas que han de dar cabida a unos contenidos mucho más amplios para cubrir a las competencias que el alumno debe adquirir.

La Orden ECl/3855/2007, por la que se establecen los requisitos para la verificación de los títulos universitarios oficiales que habiliten para el ejercicio de la profesión de Arquitecto Técnico (Orden ECl) (O.ECl, de 27 de diciembre), establece las competencias que el estudiante debe adquirir y entre estas se encuentra en su artículo 3 apartado 2 la de "Redactar estudios y planes de seguridad y salud laboral y coordinar la actividad de las empresas en materia de seguridad y salud laboral en obras de construcción, tanto en fase de proyecto como de ejecución".

La adquisición de estas competencias, dando cumplimiento a lo establecido en el Real Decreto 1627/1997, por el que se establecen disposiciones mínimas de seguridad y de salud en las obras de construcción (RD 1627/97, de 24 de octubre), conlleva un conocimiento profundo del proceso constructivo y de los procedimientos preventivos a aplicar en dicho proceso. Es por ello que la formación que reciben estos técnicos excede en gran medida de la contenida en el Segundo ciclo de formación en materia de Prevención de Riesgos Laborales del Sector de la Construcción: Formación por puesto de trabajo, y en el Nivel básico de prevención en construcción.

\section{Objetivos}

Se busca poder trasladar información relevante, a todas las escuelas que imparten los grados universitarios habilitantes para el ejercicio profesional de la Arquitectura Técnica, que permita realizar una propuesta conjunta, para el reconocimiento de la formación en materia de prevención de riesgos en obras de construcción conforme al CCGSC. 


\section{Desarrollo}

\section{1 Ámbito académico}

El Arquitecto Técnico ocupa hoy, por razón de los conocimientos adquiridos en la carrera, un papel muy importante en el sector de la edificación como generalista que conoce y aplica las técnicas al proceso constructivo.

La vinculación y el compromiso de la profesión con la Seguridad y Salud de los trabajadores en la Construcción no es reciente y es un hecho sobradamente conocido por todas las entidades y organismos relacionadas con el sector. Quizás sea fruto de esta circunstancia, amén de la especial preparación profesional de los arquitectos técnicos, el hecho de que la mayoría de los "Coordinadores de Seguridad y Salud" ejercientes en las obras de edificación en España sean Arquitectos Técnicos (Merino, 2012).

Los conocimientos que en materia de seguridad y salud adquieren los Arquitectos Técnicos en su carrera se formulan como competencias en la Orden ECl, la cual establece en su artículo 3 Objetivos, las competencias que los estudiantes deben adquirir:

1. Dirigir la ejecución material de las obras de edificación, de sus instalaciones y elementos, llevando a cabo el control cualitativo y cuantitativo de lo construido mediante el establecimiento y gestión de los planes de control de materiales, sistemas y ejecución de obra, elaborando los correspondientes registros para su incorporación al Libro del Edificio. Llevar el control económico de la obra elaborando las certificaciones y la liquidación de la obra ejecutada.

2. Redactar estudios y planes de seguridad y salud laboral y coordinar la actividad de las empresas en materia de seguridad y salud laboral en obras de construcción, tanto en fase de proyecto como de ejecución.

3. Llevar a cabo actividades técnicas de cálculo, mediciones, valoraciones, tasaciones y estudios de viabilidad económica; realizar peritaciones, inspecciones, análisis de patología y otros análogos y redactar los informes, dictámenes y documentos técnicos correspondientes; efectuar levantamientos de planos en solares y edificios.

4. Elaborar los proyectos técnicos y desempeñar la dirección de obras de edificación en el ámbito de su habilitación legal.

5. Gestionar las nuevas tecnologías edificatorias y participar en los procesos de gestión de la calidad en la edificación; realizar análisis, evaluaciones y certificaciones de eficiencia energética, así como estudios de sostenibilidad en los edificios.

6. Dirigir y gestionar el uso, conservación y mantenimiento de los edificios, redactando los documentos técnicos necesarios. Elaborar estudios del ciclo de vida útil de los materiales, 
sistemas constructivos y edificios. Gestionar el tratamiento de los residuos de demolición y de la construcción.

7. Asesorar técnicamente en los procesos de fabricación de materiales y elementos utilizados en la construcción de edificios.

8. Gestionar el proceso inmobiliario en su conjunto. Ostentar la representación técnica de las empresas constructoras en las obras de edificación.

Dichas competencias quedan reguladas por la Orden ECl conforme al Plan de Estudios dentro del módulo de Gestión, donde se fijan como competencias que deben adquirirse:

- Capacidad para programar y organizar los procesos constructivos, los equipos de obra, y los medios técnicos y humanos para su ejecución y mantenimiento.

- Conocimiento del derecho de la construcción y de las relaciones contractuales que se producen en las distintas fases del proceso de edificación, así como de la legislación, reglamentación y normativas específicas de la prevención y coordinación en materia de seguridad y salud laboral en la edificación.

- Aptitud para redactar estudios, estudios básicos y planes de seguridad y salud laboral, y coordinar la seguridad en fase de proyecto o en fase de ejecución de obra.

Añadir que aun siendo este el módulo donde se abordan de una forma concreta las competencias en el ámbito de la seguridad y salud, el resto de los módulos específicos tales como el de Expresión Gráfica, Técnicas y Tecnología de la Edificación, Estructuras e Instalaciones de la Edificación, Gestión del proceso, Gestión Urbanística y Economía aplicadas y Proyectos Técnicos lo abordan de forma transversal.

\section{2 Ámbito profesional}

Profesionalmente y en relación a dichas competencias, el Arquitecto Técnico es considerado por la Ley 38/1999 de Ordenación de la Edificación (LOE) (L38/1999 de 5 de noviembre) el técnico competente al que hace mención el RD 1627/1997 conforme a lo que establece su Guía Técnica, la cual:

Considera "técnico competente" aquella persona que posee titulaciones académicas y profesionales habilitantes, así como conocimientos en actividades de construcción y de prevención de riesgos laborales acordes con las funciones a desempeñar según el RD $1627 / 1997$.

A este respecto, la disposición adicional cuarta de la LOE señala que "las titulaciones académicas y profesionales habilitantes para desempeñar la función de coordinador de seguridad y salud en obras de edificación, durante la elaboración del proyecto y la ejecución de la obra, serán las de arquitecto, arquitecto técnico, ingeniero o ingeniero técnico, de acuerdo con sus competencias y especialidades".

Para las obras de construcción excluidas del ámbito de aplicación de la LOE, así como para las obras de ingeniería civil, a los efectos de delimitar la figura de técnico competente, cabe 
interpretar que las titulaciones académicas y profesionales que habilitan para desempeñar las funciones de coordinador en materia de seguridad y salud durante la elaboración del proyecto y la ejecución de este tipo de obras serán las que estén facultadas, con arreglo a las competencias propias de sus específicas titulaciones, para proyectar y dirigir dichas obras a la vista de las disposiciones legales vigentes para cada profesión.

En base a las competencias adquiridas en el título de Grado, el Arquitecto Técnico es competente para llevar a cabo el encargo global en materia de seguridad y salud, que comprende la coordinación de seguridad durante la elaboración del proyecto, con redacción de Estudio o Estudio básico de seguridad y salud y la coordinación de seguridad en fase de ejecución de la obra, junto a la aprobación de los Planes de Seguridad y Salud en el trabajo

Como coordinación de seguridad y salud durante la elaboración del proyecto se encargará de:

- Coordinar que se tengan en cuenta los principios generales de prevención en materia de seguridad y salud durante las fases de concepción, estudio y elaboración del proyecto y las previsiones contenidas en el Estudio o en el Estudio Básico de seguridad y salud. Y elaborar o hacer que se elabore, bajo su responsabilidad, el Estudio o Estudio Básico de seguridad y salud.

Como coordinación en materia de seguridad y salud durante la ejecución de la obra sus funciones son:

- Coordinar la aplicación de los principios generales de prevención.

- Coordinar las actividades a desarrollar en la obra para garantizar que se aplique la acción preventiva por parte de las empresas intervinientes, así como de los trabajadores autónomos.

- Aprobar el Plan de seguridad y salud en el trabajo.

- Organizar la coordinación de las actividades empresariales.

- Coordinar la aplicación correcta de los métodos de trabajo.

- Adoptar medidas de control de acceso a la obra.

- Ser depositario del Libro de Incidencias y hacer anotaciones en el mismo.

- Formular al contratista instrucciones en materia de seguridad y salud.

- Remitir copia de las anotaciones del Libro de Incidencias a la Inspección Provincial de Trabajo y Seguridad Social.

- Paralizar los trabajos en caso de observarse incumplimiento de las medidas de seguridad y salud. 
Sirve todo lo anterior para fijar los contenidos que, desde las distintas Universidades, y para los Grados que habilitan para el ejercicio de la profesión de Arquitecto Técnico, se imparten como asignatura troncal de al menos 6 ECTS (60 horas presenciales, equivalentes a 150 horas de trabajo del alumno), llegando a alcanzar los 9 ECTS (90 horas presenciales, equivalentes a 225 horas de trabajo del alumno) en la mayoría de las Escuelas, tal y como puede comprobarse en los planes de estudio.

\subsection{Contenidos conforme al Convenio}

El Capítulo III del VI CGSC relativo a la formación desarrolla, en su Sección Primera y Segunda a través de los artículos 139 y 140, el Segundo ciclo de formación en materia de prevención de riesgos laborales: formación por puesto de trabajo y el Nivel básico de prevención en la construcción. Dicha formación en relación con la formación inicial, los puestos de trabajo de personal directivo, responsables de obra y técnicos de ejecución, mandos intermedios, delegados de prevención y nivel básico de prevención en construcción, desarrollan contenidos que pueden ser considerados equivalentes a los impartidos en los títulos universitarios oficiales que habilitan para el ejercicio de la profesión de Arquitecto Técnico.

Si marcamos en dichos contenidos los no comunes entre sí, tal y como aparecen en la tabla 1 , podemos determinar cuáles son los epígrafes a desarrollar. 
Convalidación de la formación en materia de Prevención de Riesgos Laborales del Sector de la Construcción establecida en el Convenio a los titulados de las Escuelas de Edificación y Arquitectura

Técnica de España - Validation of the training in Occupational Risk Prevention in the Construction Sector established in the Agreement for graduates of the Spanish Schools of Building and Technical Architecture.

\section{Tabla 1. Contenidos formación anexo XII VI CGSC}

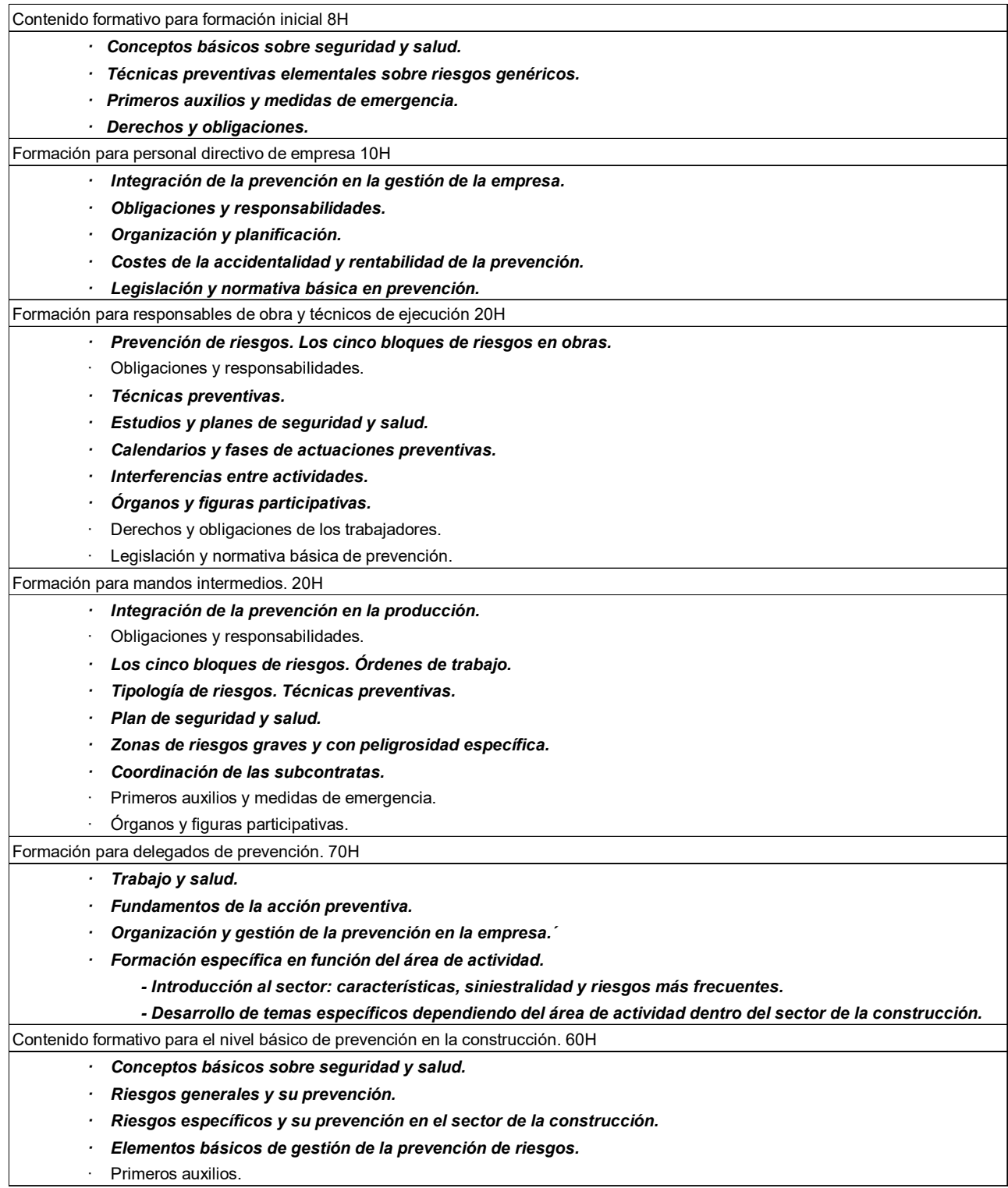


Los epígrafes serían:

- Conceptos básicos sobre seguridad y salud. (Trabajo y salud, fundamentos de la acción preventiva)

- Técnicas preventivas elementales sobre riesgos genéricos.

- Primeros auxilios y medidas de emergencia.

- Derechos y obligaciones.

- Integración de la prevención en la gestión de la empresa.

- Obligaciones y responsabilidades.

- Organización y planificación.

- Costes de la accidentalidad y rentabilidad de la prevención.

- Legislación y normativa básica en prevención.

- Prevención de riesgos. Los cinco bloques de riesgos en obras.

- Técnicas preventivas.

- Estudios y planes de seguridad y salud.

- Calendarios y fases de actuaciones preventivas.

- Interferencias entre actividades.

- Órganos y figuras participativas.

- Los cinco bloques de riesgos. Órdenes de trabajo.

- Tipología de riesgos.

- Zonas de riesgos graves y con peligrosidad específica.

- Coordinación de las subcontratas.

- Organización y gestión de la prevención en la empresa.

- Formación específica en función del área de actividad.

- Riesgos generales y su prevención.

- Riesgos específicos y su prevención en el sector de la construcción.

- Elementos básicos de gestión de la prevención de riesgos.

Todos y cada uno de estos epígrafes, son objeto de desarrollo en los contenidos impartidos en los títulos universitarios oficiales que habilitan para el ejercicio de la profesión de Arquitecto Técnico, tal y como puede comprobarse en las guías docentes de las asignaturas correspondientes a la materia de prevención y seguridad.

\section{Resultado y conclusiones}

El Arquitecto Técnico asume como técnico competente actuaciones en materia de seguridad y salud que conllevan la necesidad de tener un conocimiento profundo del proceso productivo y preventivo.

Esta necesidad se contempla tanto el la Orden $\mathrm{ECl}$, donde se establecen las competencias en la materia, como en los planes de estudios, donde se incluyen créditos en asignaturas troncales donde se imparten los contenidos de la materia de prevención y seguridad y se asumen dichas competencias.

Profesionalmente se asumen responsabilidades que sólo desde un conocimiento profundo del proceso productivo pueden abordarse. Conocimientos que los profesionales de la Arquitectura Técnica poseen en mayor nivel que los Titulados de formación profesional o los adquiridos en Certificados de profesionalidad 
Convalidación de la formación en materia de Prevención de Riesgos Laborales del Sector de la Construcción establecida en el Convenio a los titulados de las Escuelas de Edificación y Arquitectura

Técnica de España - Validation of the training in Occupational Risk Prevention in the Construction

Sector established in the Agreement for graduates of the Spanish Schools of Building and Technical Architecture.

No se trata de cuestionar si los contenidos impartidos son los mismos que los que marca el CCGSC sino de reconocer que la formación recibida excede de la requerida.

Vistas las competencias de la Orden $\mathrm{ECl}$, los créditos asignados para su adquisición en los planes de estudio, la actividad relacionada en el ejercicio profesional, así como los contenidos que se establecen en el CCGSC, la formación en materia de prevención de riesgos en obras de construcción de los Arquitectos Técnicos debe poder obtener el reconocimiento.

Según el artículo 146 del Convenio General del Sector de la Construcción, sobre formación impartida en Títulos Universitarios, en relación con los trabajadores que estén en posesión de títulos universitarios en los que se imparta una formación en materia de prevención de riesgos laborales equivalente a la especificada en el presente Convenio, se procederá a su convalidación, según proceda en cada caso, mediante acuerdo del Patronato de la FLC.

EI Anexo XIII del CGCC en su apartado 2 recoge un cuadro resumen de reconocimientos con Títulos de formación profesional y Certificados de profesional en las ramas de edificación y obra civil.

Dicho reconocimiento puede por tanto realizarse por la vía de un acuerdo por el patronato, o por la del establecimiento de dichos reconocimientos de forma directa en el Convenio, siendo este último el medio mediante el cual deberían poder incluirse todas las titulaciones universitarias oficiales que habilitan para el ejercicio de la profesión de Arquitecto Técnico.

\section{Referencias}

España. Ley 38/1999, de 5 de noviembre, de Ordenación de la Edificación. BOE, 6 de noviembre de 1999, núm. 266, p. 38925 a 38934.

España. Orden ECI/3855/2007, de 27 de diciembre, por la que se establecen los requisitos para la verificación de los títulos universitarios oficiales que habiliten para el ejercicio de la profesión de Arquitecto Técnico. BOE, 29 de diciembre de 2007, núm. 312, p. 53739 a 53742.

España. Real Decreto 1627/1997, de 24 de octubre, por el que se establecen disposiciones mínimas de seguridad y de salud en las obras de construcción. BOE, 25 de octubre de 1997, núm. 256, p.30875 a 30886 .

España. Resolución de 21 de septiembre de 2017, de la Dirección General de Empleo, por la que se registra y publica el Convenio colectivo general del sector de la construcción. BOE, 26 de septiembre de 2017, núm. 232, p. 94090 a 94253.

MERINO VELASCO, F. (2012). Competencias y atribuciones del arquitecto técnico en Europa : estudio específico en Italia, Austria y Polonia. Trabajo Final de Grado. Valencia: Universitat Politècnica de València, < https://riunet.upv.es/handle/10251/17769> [Consulta: 1 de mayo 2021] 